\title{
sciendo
}

\section{Characterization of the Weekly External Load Profile of Professional Soccer Teams From Portugal and the Netherlands}

\author{
by \\ Filipe Manuel Clemente ${ }^{1,2}$, Adam Owen ${ }^{3,4}$, Jaime Serra-Olivares ${ }^{5}$, \\ Pantelis Theodoros Nikolaidis ${ }^{6}$, Cornelis M. I. van der Linden ${ }^{7}$, Bruno Mendes ${ }^{4}$
}

The purpose of this study was to analyze the day-to-day variance of a typical weekly external training workload of two professional soccer teams from different countries. Twenty-nine players from two professional teams from Portugal and the Netherlands participated in this study. The players' external load was monitored for 7 weeks, by means of portable GPS devices (10 Hz, JOHAN, Noordwijk, Netherlands). Results revealed that match day -1 (MD-1), i.e. the training day before a match, had significantly $(p=0.001)$ less training volume $(4584.50 \mathrm{~m})$ than the other days. $M D-5$ (training five days before a match), MD-4 (four days before a match) and MD-3 (three days before a match) were the most intense (390.83, 176.90 and $247.32 \mathrm{~m}$ of sprinting distance, respectively) and with large volume (7062.66, 6077.30 and $6919.49 \mathrm{~m}$, respectively). Interestingly, significant differences were found between clubs of different countries ( $p$ <.05) with the Portuguese team showing significantly higher intensity (sprinting distance) and volume (total distance) in all days with exception of MD-1 than the Dutch team. The results of this study possibly allow for the identification of different training workloads and tapering strategies between countries in relation to volume and intensity. It should be noted, however, that both clubs used a significant tapering phase in the last two days before the competition in an attempt to reduce residual fatigue accumulation.

Key words: soccer, workload, microcycle, external load, GPS, tapering.

\section{Introduction}

Team sports such as soccer imply producing repeatedly high-speed movements and directional changes with brief recovery periods (Buchheit et al., 2010; Dellal et al., 2010). Soccer players suffer from longitudinal fixture congestion across the season, and muscle soreness and fatigue are very common during week training seasons (Owen et al., n.d.; Rampinini et al., 2011). For these reasons, soccer coaching requires the adaptation of training loads for performance improvement (Foster et al., 2001; Issurin, 2010; Manzi et al., 2010).
Previous research has been conducted on the volume and intensity variations in soccer training sessions and microcycles (Clemente et al., 2017; Clemente and Nikolaidis, 2016; Coutinho et al., 2015; Owen et al., 2017). The existing literature shows that monitoring training and weekly sessions is suggested as a key to understand the demands variations (Gamble, 2013, 2012). However, there is no protocol showing how to adapt the training loads in elite soccer during the week. Previous research is also inconclusive about the discrepancies on how to manage the training loads during the week in soccer (Los Arcos et al.,

1 - Instituto Politécnico de Viana do Castelo, Escola Superior de Desporto e Lazer, Portugal.

2 - Instituto de Telecomunicações, Portugal.

3 - Centre de Recherche et d'Innovation sur le Sport, Université Claude Bernard Lyon.1, Lyon, France.

4 - Benfica Lab, Sport Lisboa e Benfica, Portugal.

5 - Pedagogy in Physical Education. Faculty of Education. Catholic University of Temuco, Chile.

6 - Exercise Physiology Laboratory, Nikaia, Greece.

7 - JOHAN sports, Department of Sport Sciences, Netherlands. 
2017; Malone et al., 2016). As a result, further analysis concerning how technical and physical staff manage and monitor players' training loads is warranted.

Recent literature concerning the use of soccer training monitoring methods includes the analysis of key variables assessed such as distance covered, speed, acceleration or players body impacts using Global Positioning System (GPS) devices (Cummins et al., 2013; Dwyer and Gabbett, 2012). Their findings have confirmed differences between players in some time motion variables such as the number of sprints performed, high-intensity movements or the total distance covered, depending on the competition type (Casamichana et al., 2013), players' positions (Bradley and Noakes, 2013; Clemente et al., 2013; Di Salvo et al., 2007), the playing level (Mohr et al., 2003; Rampinini et al., 2007) or age (Abade et al., 2014; Mendez-Villanueva et al., 2012).

Some researchers have analyzed the day-today differences within the microcycle training loads (Anderson et al., 2016; Malone et al., 2015). It seems that training loads are higher in the first days of the microcycle. Lighter loads tend to be used in a tapering strategy approximately $48 \mathrm{hrs}$ pre-match (Anderson et al., 2016; Casamichana et al., 2013; Jeong et al., 2011; Wrigley et al., 2012). Such a progressive decrement of individual responses to training observed as the competition days approach, further promotes a conscious and precompetitive unloading strategy (Bosquet et al., 2007; Impellizzeri et al., 2004).

Previous research has shown higher intensities in the 48 hours post-match in youth soccer players (Coutinho et al., 2015). In the same way, high external loads have been detected in the days that are far from the match (Stevens et al., 2017). Therefore, it appears that coaches manage players' loads in training by reducing the total distance (volume) and high sprint running (intensity) in the days preceding a match (Akenhead et al., 2016; Anderson et al., 2016). Only these few studies have analyzed the quantification of weekly loads focusing on differences between daily training loads. Therefore, it remains unclear yet which training strategy would be better to use depending on the competition schedule and player's efforts (Fessi et al., 2016). There is a need to understand the weekly distribution of training loads, that is even more important during the competitive season (Impellizzeri et al., 2004; Owen and Wong, 2009). The characterization of the weekly training profile of soccer teams and comparison between different countries may be important to identify whether significant changes occur or whether the training process is similar across different professional realities. This comparison will help in benchmarking the training load profile. Based on that, the purpose of this study was to analyze the variance of typical weekly external workloads of two professional soccer teams from different countries and compare the training load between days of a week. A 7-week study during the middle of the 2016-2017 season was conducted, monitoring the variables of total distance, walking distance, jogging distance, running distance, sprint distance, maximum speed, the number of sprints per minute and pace during all training sessions of one professional club from Portugal and one from the Netherlands.

\section{Methods}

Participants

Twenty-nine professional soccer players from two professional teams participated in this study: one from the Portuguese Second League (n $=14$, age $19.21 \pm 1.05$ yrs; body height $180.14 \pm$ $5.96 \mathrm{~cm}$; body mass $74.07 \pm 6.21 \mathrm{~kg}$ ) and one from the Dutch Second League $(n=15$, age 25.14 \pm 3.90 yrs; body height $179 \pm 6.06 \mathrm{~cm}$; body mass 73.21 $\pm 6.46 \mathrm{~kg}$ ). Players were classified by their typical playing position: i) defenders (DFs); ii) midfielders (MFs); iii) forwards (FWs). Ten DFs, nine MFs and ten FWs were monitored during seven consecutive weeks (November 2016 to January 2017). All the participants were informed about the study protocol and signed an informed consent form. The experiment followed the ethical standards of the Declaration of Helsinki for research in humans.

Design

Training data were collected over a 7-week period during the middle of the 2016-2017 season. During this period, players had one official match in the weekends. The days of training were classified as +1 and +2 (match days: MD) and $<-5$, $-5,-4,-3,-2$ and -1 days before a match. For each week, external load data of players who did not participate in all training sessions were excluded from the analysis. Goalkeepers were not included 
in the analysis. During the investigation, the players completed 5/6 training sessions across 6 days. In both Portuguese and Dutch teams, a recovery day-off was conducted the day after the match (MD+1).

\section{External load}

The workload was measured by a geolocation tracker (JOHAN Sports, Noordwijk, The Netherlands), consisting of a GPS sensor (10 $\mathrm{Hz}$, including EGNOS correction), Accelerometer, Gyroscope \& Magnetometer $(100 \mathrm{~Hz}, 3$ axis, \pm 16 g). The value of $10 \mathrm{~Hz}$ seems to be valid and reliable enough to measure the position and speed in a sport setting (Scott et al., 2016). The GPS sensor used in this study was tested with $2.5 \pm$ $0.41 \%$ (error \pm deviation) reliability for total distance covered (Clemente et al., 2017).

Players were firstly familiarized with GPS trackers prior to the experiment. The weather conditions during the weeks varied, however, not compromising the GPS reception. Players wore a body tight vest to ensure valid (e.g. body oriented) accelerometer data. A motion tracker was then placed in a bag of the vest located in the dorsal region of the players. After each training session, motion data from the trackers were uploaded in the JOHAN Sports online analysis platform.

Distance covered in the different speed thresholds was measured according to the following classification: i) total distance (m); ii) walking distance $(0-6.9 \mathrm{~km} / \mathrm{h})$; iii) jogging distance $(7.0-13.9 \mathrm{~km} / \mathrm{h})$; iv) running distance (14.0 - $20.0 \mathrm{~km} / \mathrm{h}) ; \mathrm{v})$ sprint distance $(>20.0 \mathrm{~km} / \mathrm{h})$. Maximum speed $(\mathrm{km} / \mathrm{h})$, the number of sprints per minute $(\mathrm{n} / \mathrm{min})$ and pace $(\mathrm{m} / \mathrm{min})$ were also estimated by the GPS tracker. The player load (g/min) was provided with support of an accelerometer. This variable is an accumulation of data collected from all axes (anteroposterior, mediolateral and craniocaudal) (Clemente et al., 2017).

Statistical Analysis

The influences of the day of training and the country of the team on the dependent variables from GPS were analyzed using multiavriate MANOVA after validating normality and homogeneity assumptions. MANOVA was chosen based on the fact that it reduces Type I Error Inflation compared with ANOVA (O'Donoghue, 2012). When MANOVA detected significant statistical differences between the two factors, we proceeded to the two-way ANOVA for each dependent variable, followed by the Tukey's HSD post-hoc test (O'Donoghue, 2012). If no interactions were detected in two-way ANOVA, one-way ANOVA was used for each independent variable. The partial eta squared $\left(\eta_{p}^{2}\right)$ tested the effect size (ES). The Ferguson's classification for the ES was used (Ferguson, 2009): no effect (ES < $0.04)$; minimum effect $(0.04<\mathrm{ES}<0.25)$; moderate effect $(0.25<\mathrm{ES}<0.64)$; and strong effect (ES > $0.64)$. The variance between both tests was estimated with an independent t-test followed by Cohen's $d$ to analyze the effect size. The following classification to measure the magnitude of ES was used (Ferguson, 2009): no effect $(d<0.41)$, minimum effect $(0.41<d<1.15)$, moderate effect $(1.15<d<2.70)$ and strong effect $(d>2.70)$. All statistical analysis was carried out using SPSS statistical analysis software (SPSS version 23.0, Chicago, USA). The level of statistical significance was set at $p \leq 0.05$.

\section{Results}

Multivariate MANOVA revealed that the country of the team $\left(p=0.001 ; \eta_{p}^{2}=0.210\right)$ and day after a match $\left(p=0.001 ; \eta_{p}^{2}=0.144\right)$ had significant main effects on the composite of the external load. There was significant interaction (Pillai's Trace $=$ $0.674 ; p=0.001 ; \eta_{p}^{2}=0.096$ ) between the country of the team and the day after a match on the external load. The descriptive statistics of dependent variables can be found in Figures 1 and 2 .

The independent $t$-test estimated the variance of the dependent variable between teams per day of the week. In MD+1 significantly greater values of total distance $(p=0.012 ; d=3.74)$, walking distance $(p=0.004 ; d=5.23)$ and running distance $(p=0.046 ; d=1.81)$ were found in the Portuguese team. In MD+2 significantly greater values of total distance $(p=0.001 ; d=1.92)$, jogging distance $(p=0.001 ; d=1.71)$, sprinting distance $(p=0.001 ; d=0.84)$, player load $(p=0.005$; $d=1.78)$ and the number of sprints $(p=0.001 ; d=$ $0.57)$ were found in the Portuguese team. In MD-3 significantly greater values of total distance $(p=$ $0.001 ; d=1.93)$, walking distance $(p=0.003 ; d=$ $1.70)$, jogging distance $(p=0.001 ; d=1.15)$, running distance $(p=0.001 ; d=1.15)$, sprinting distance $(p$ $=0.002 ; d=1.66)$, maximum speed $(p=0.030 ; d=$ 1.14), pace $(p=0.021 ; d=0.76)$, 
and the number of sprints per minute $(p=0.001 ; d$ $=1.48$ ) were found in the Portuguese team. However, in MD-3 significantly greater value of player load ( $p=0.005 ; d=1.18$ ) was found in the Dutch team.

In MD-2 significantly greater values of total distance $(p=0.001 ; d=0.76)$, walking distance $(p=0.001 ; d=1.09)$, running distance ( $p=$ $0.001 ; d=0.95)$, sprinting distance $(p=0.001 ; d=$ $0.89)$, maximum speed ( $p=0.001 ; d=0.62)$, and the number of sprints per minute $(p=0.001 ; d=0.60)$ were found in the Portuguese team. Finally, in MD-1 significantly greater values of total distance $(p=0.001 ; d=0.84)$, jogging distance $(p=0.001 ; d=$ $4.49)$, sprinting distance $(p=0.023 ; d=0.44)$, pace $(p=0.001 ; d=0.99)$, player load $(p=0.001 ; d=2.09)$ and the number of sprints per minute $(p=0.010 ; d$ $=0.65$ ) were found in the Dutch team.

Significant differences were found in total distance $\left(p=0.001 ; \eta^{2}=0.343\right)$, walking distance $(p$ $\left.=0.001 ; \eta^{2}=0.195\right)$, jogging distance $\left(p=0.001 ; \eta^{2}\right.$ $=0.432)$, running distance $\left(p=0.001 ; \eta^{2}=0.311\right)$, sprinting distance $\left(p=0.001 ; \eta^{2}=0.147\right)$, maximum speed $\left(p=0.001 ; \eta^{2}=0.077\right)$, pace $(p=$ $\left.0.001 ; \eta^{2}=0.357\right)$, player load $\left(p=0.001 ; \eta^{2}=\right.$ $0.272)$ and the number of sprints per minute $(p=$ $0.001 ; \eta^{2}=0.088$ ) between days of the week. The post hoc differences are presented in Table 1.

Table 1

Descriptive statistics (mean \pm SD) of the total external load of both teams and post hoc analysis.

\begin{tabular}{|c|c|c|c|c|c|c|c|}
\hline & +1 & +2 & -5 & -4 & -3 & -2 & -1 \\
\hline $\begin{array}{l}\text { Total } \\
\text { distance } \\
(\mathrm{m})\end{array}$ & $\begin{array}{c}6023.05 \pm 169 \\
1.25\end{array}$ & $\begin{array}{c}6277.06 \pm 1422 \\
77^{\mathrm{e}, \mathrm{g}}\end{array}$ & $\begin{array}{c}7062.66 \pm 1460 \\
.61^{\mathrm{g}}\end{array}$ & $\begin{array}{c}6077.30 \pm 10 \\
54.76\end{array}$ & $\begin{array}{c}6919.49 \pm 1846 \\
.33^{\mathrm{b}, \mathrm{f}, \mathrm{g}}\end{array}$ & $\begin{array}{c}5701.57 \pm 12 \\
72.49^{\mathrm{e}, \mathrm{g}}\end{array}$ & $\begin{array}{c}4584.50 \pm 1053.1 \\
1^{\mathrm{b}, \mathrm{c}, \mathrm{e}, \mathrm{f}}\end{array}$ \\
\hline $\begin{array}{l}0-6.9 \mathrm{~km} / \mathrm{h} \\
(\mathrm{m})\end{array}$ & $\begin{array}{c}2852.62 \pm 700 \\
31\end{array}$ & $\begin{array}{c}2741.51 \pm 315.6 \\
6^{\mathrm{e}, \mathrm{f}}\end{array}$ & $\begin{array}{c}3066.75 \pm 717 \\
69\end{array}$ & $\begin{array}{c}2995.42 \pm 35 \\
7.05^{\mathrm{e}}\end{array}$ & $\begin{array}{c}3552.52 \pm 1154 \\
.94^{\mathrm{b}, \mathrm{d}, \mathrm{f}, \mathrm{g}}\end{array}$ & $\begin{array}{c}3063.42 \pm 59 \\
1.53^{\mathrm{b}, \mathrm{e}, \mathrm{g}}\end{array}$ & $\underset{\mathrm{e}, \mathrm{f}}{2719.82 \pm 678.63}$ \\
\hline $\begin{array}{l}7-13.9 \mathrm{~km} / \mathrm{h} \\
(\mathrm{m})\end{array}$ & $\begin{array}{c}2501.20 \pm 884 . \\
80 \mathrm{~g}\end{array}$ & $\begin{array}{c}2824.75 \pm 1081 \\
93^{\mathrm{f}, \mathrm{g}}\end{array}$ & $\begin{array}{c}2614.67 \pm 719 \\
96^{\mathrm{f}, \mathrm{g}}\end{array}$ & $\begin{array}{c}2381.68 \pm 89 \\
8.51 \mathrm{~g}\end{array}$ & $\begin{array}{c}2496.53 \pm 493 . \\
46^{\mathrm{f}, g}\end{array}$ & $\begin{array}{c}1859.68 \pm 54 \\
9.61^{\mathrm{b}, \mathrm{c}, \mathrm{e}, \mathrm{g}}\end{array}$ & $\begin{array}{c}1378.77 \pm 554.67 \\
\mathrm{a}, \mathrm{b}, \mathrm{c}, \mathrm{d}, \mathrm{d}, \mathrm{f}, \mathrm{f}\end{array}$ \\
\hline $\begin{array}{l}14-20 \mathrm{~km} / \mathrm{h} \\
(\mathrm{m})\end{array}$ & $\begin{array}{c}513.62 \pm 228.8 \\
0\end{array}$ & $573.13 \pm 371.49^{c}$ & $\begin{array}{c}990.43 \pm 359.6 \\
7^{\mathrm{b}, \mathrm{f}, \mathrm{g}}\end{array}$ & $\begin{array}{c}523.29 \pm 181 \\
70\end{array}$ & $\begin{array}{c}623.13 \pm 268.8 \\
7 \mathrm{~g}\end{array}$ & $\begin{array}{c}532.20 \pm 279 \\
.45^{\mathrm{c}, \mathrm{g}}\end{array}$ & $\underset{\mathrm{e}, \mathrm{f}}{342.92 \pm 172.20^{\mathrm{c}}}$ \\
\hline $\begin{array}{l}>20 \mathrm{~km} / \mathrm{h} \\
(\mathrm{m})\end{array}$ & $155.62 \pm 83.72$ & $137.67 \pm \underset{e, f}{1118.05^{c}}$ & $\begin{array}{c}390.83 \pm 259.4 \\
6^{\mathrm{b}, \mathrm{g}}\end{array}$ & $\begin{array}{c}176.90 \pm 82.0 \\
9\end{array}$ & $\begin{array}{c}247.32 \pm 239.6 \\
4^{\mathrm{b}, \mathrm{g}}\end{array}$ & $\begin{array}{c}246.29 \pm 200 \\
.28^{\mathrm{b}, \mathrm{g}}\end{array}$ & $142.99 \pm \underset{e, f}{117.17}$ \\
\hline $\begin{array}{l}\text { Max speed } \\
(\mathrm{km} / \mathrm{h})\end{array}$ & $27.42 \pm 2.79$ & $25.76 \pm 3.55^{\mathrm{e}, \mathrm{f}}$ & $29.93 \pm 2.27$ & $27.09 \pm 2.62$ & $29.42 \pm 8.23^{\mathrm{b}}$ & $28.33 \pm 6.03^{b}$ & $27.24 \pm 3.44$ \\
\hline $\begin{array}{l}\text { Pace } \\
(\mathrm{m} / \mathrm{min})\end{array}$ & $83.98 \pm 11.07$ & $87.81 \pm 19.44^{\mathrm{c}, \mathrm{fg} g}$ & $\underset{, \mathrm{d}, \mathrm{e}, \mathrm{f}, \mathrm{g}}{106.17 \pm 18.12^{\mathrm{b}}}$ & $80.70 \pm \underset{c}{ \pm} 13.44$ & $83.12 \pm 8.35^{\mathrm{c}, \mathrm{fg} g}$ & $\begin{array}{c}73.21 \pm 12.4 \\
2^{\mathrm{b}, \mathrm{c}, \mathrm{e}, \mathrm{g}}\end{array}$ & $65.89 \pm 14.88^{\mathrm{b}, c, \mathrm{e}, \mathrm{f}}$ \\
\hline $\begin{array}{l}\text { Player } \\
\text { Load } \\
\text { (g/min) }\end{array}$ & $4.43 \pm 0.79$ & $4.18 \pm 0.78^{\mathrm{f}, \mathrm{g}}$ & $5.09 \pm 1.09^{\mathrm{e}, \mathrm{f}, \mathrm{g}}$ & $4.30 \pm 1.20 \mathrm{~g}$ & $4.19 \pm 0.67^{c, g}$ & $3.70 \pm 0.83^{\mathrm{b}, \mathrm{c}}$ & $3.50 \pm 0.97 \mathrm{~b}, c, \mathrm{~d}, \mathrm{e}$ \\
\hline $\begin{array}{l}\text { Number of } \\
\text { sprints } \\
(\mathrm{n} / \mathrm{min})\end{array}$ & $0.05 \pm 0.05$ & $0.03 \pm 0.05^{c, f}$ & $0.12 \pm 0.11^{\mathrm{b}, \mathrm{g}}$ & $0.05 \pm 0.05$ & $0.05 \pm 0.05$ & $0.06 \pm 0.07^{\mathrm{b}}$ & $0.04 \pm 0.05^{c}$ \\
\hline
\end{tabular}

Significantly different from $+1^{a} ;+2^{b} ;-5 c ;-4^{d} ;-3 e ;-2 f ;-18$ for a $p<0.05$ 

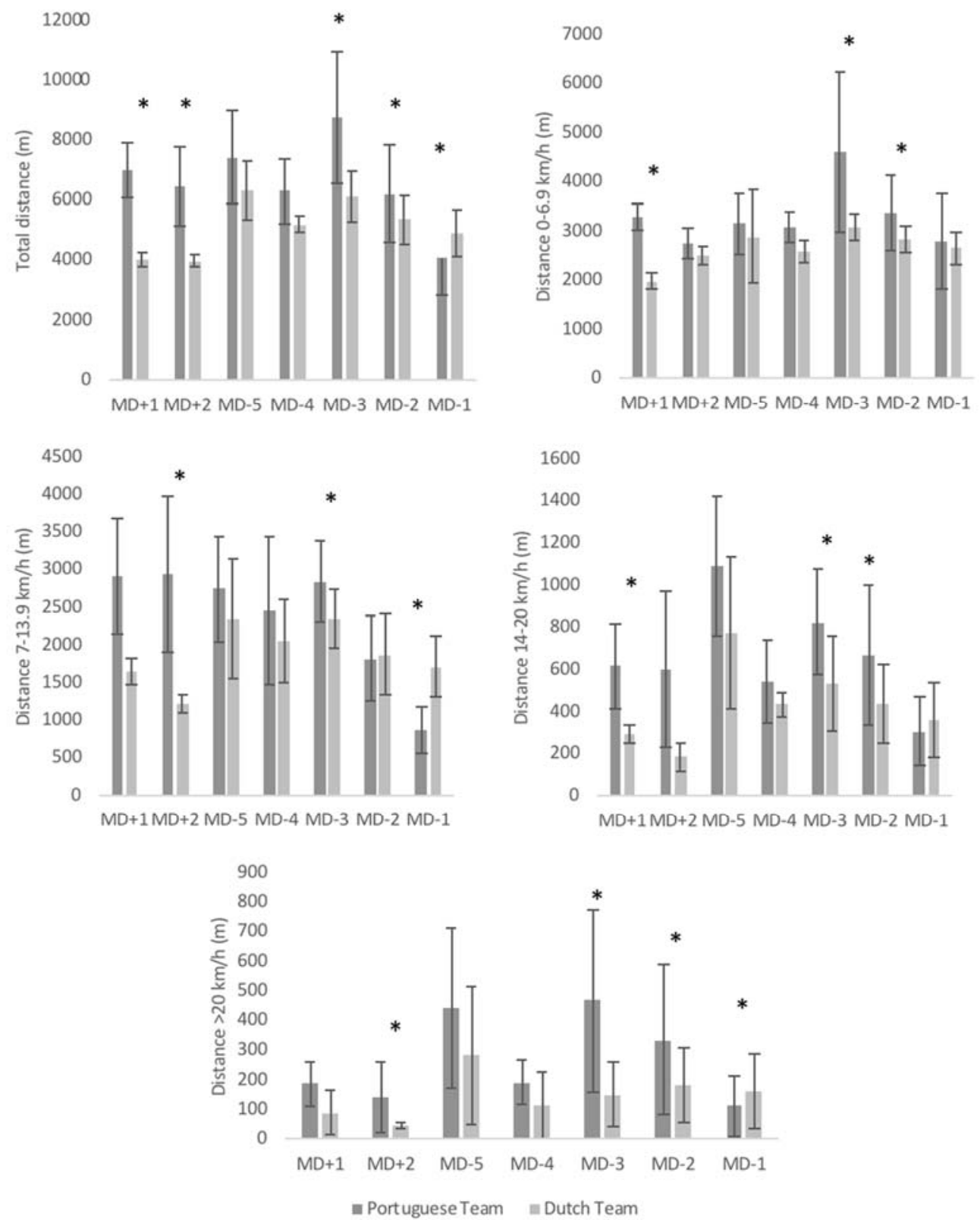

Figure 1

Descriptive statistics (mean $\pm S D$ ) of distances covered at different speeds between teams and days of the week. ${ }^{*}$ Significant difference between teams at $p<0.05$ 


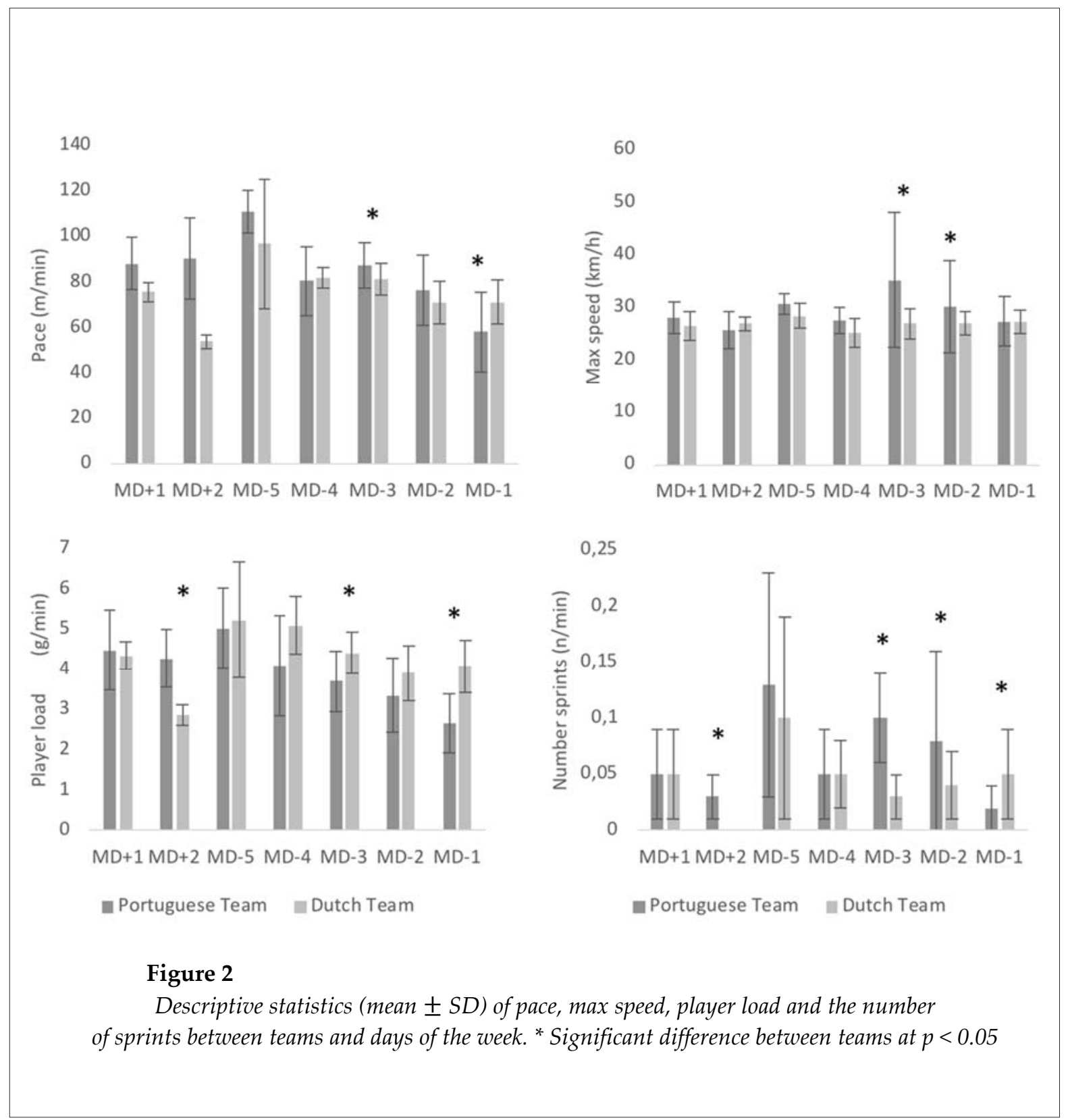

\section{Discussion}

The primary aim of this investigation was to compare the external training load of two professional soccer teams of a similar level from different countries which were considered to be among the best in soccer in the world. Additionally, the intra-week variance was also tested in order to identify the load oscillation during the training week. Results from the study confirmed that significant differences of the external load between teams existed. The team from Portugal covered more distance in different speed zones with the exception of MD-1. The intra-week comparison revealed that $\mathrm{MD}+2, \mathrm{MD}-$ 5, MD-4 and MD-3 had greater loads than MD+1, MD-2 and MD-1.

A recent study conducted amongst elite youth Portuguese players revealed that U19 age groups performed more sprints and covered more total distance at various intensities during the post-match period than during the middle of the week or pre-match days (Coutinho et al., 2015). In this case, the specific small-sided games played at large fields may have influenced such results (Coutinho et al., 2015). In a study conducted in 
Dutch teams, the regular training days were compared with the match load in players from the Eredivisie team (Stevens et al., 2017). Training on MD-4 had higher values of total distance and high sprint distance; furthermore, a progressive decrease in the values until MD-1 was observed (Stevens et al., 2017). Our results are in line with previous research (Coutinho et al., 2015; Stevens et al., 2017), showing a progressive decrease of the load from the middle of the week until MD-1. Results of our study revealed that greater distance was covered in days MD-5 and MD-3 and greater sprint distances were covered between MD- 5 and MD-2. Interestingly, MD-2 had a smaller load than MD-5, MD-4 and MD-3 (distance covered, player load), but similar high intensity distances, thus suggesting a decrease in the volume, while maintaining intensity. The decrease in the volume during the last two days before the match can be associated with the intra-week tapering period conducted to diminish fatigue, recover from the middle training sessions' load and increase the chances of high performance in the match (Impellizzeri et al., 2004; Owen and Wong, 2009). This evidence has been confirmed in recent studies conducted on soccer that found significant decreases in total distance, high sprint distances, the amount of acceleration and deceleration performed in the last two days before the match (Akenhead et al., 2016; Anderson et al., 2016).

Results from our study revealed greater volumes of total distance achieved $\sim 7000 \mathrm{~m}$, of which $990 \mathrm{~m}$ were covered at high running speed and $390 \mathrm{~m}$ at sprint speed. These high sprint results are slightly greater than the findings in Dutch teams ( $281 \mathrm{~m}$ of high sprint running) (Stevens et al., 2017) and English teams (100 to 190 $\mathrm{m}$ of high sprint running) (Akenhead et al., 2016; Anderson et al., 2016). Specifically, the team analyzed from Portugal covered $444 \mathrm{~m}$ at more than $20 \mathrm{~km} \cdot \mathrm{h}^{-1}$, thus representing $\sim 56 \%$ more distance at this intensity than the team from the Netherlands. These high levels of high-intensity exercise can be justified by the specific training methodology in Portuguese teams that tends to use many competitive small-sided games, increasing the individual participation of players and their effort during the different formats of play (Coutinho et al., 2015).

The comparison between teams from different countries also revealed interesting evidence: two days after the match had significantly more volume in the Portuguese team and the day before the match had significantly more volume in the Dutch team. The Portuguese team covered $\sim 73 \%$ and $\sim 62 \%$ more distance than the Dutch team in the first and second days after the match, respectively. No significant differences were found in MD-5 and MD-4 between teams. In the day before the match it was found that the Dutch team covered more $(\sim 20 \%)$ distance than the Portuguese team. This methodological options lead to different strategies for the microcycle. The Portuguese team opt to use a greater volume during training sessions that is significantly reduced on the last day, thus being a clear tapering day. Such a strategy is not so clear in the Dutch team.

Interestingly, the number of sprints performed by players during the training sessions is also different between the Portuguese and Dutch team. The Portuguese team completed more sprints on MD-5, MD-3 and MD-2 (0.08 to $0.013 \mathrm{n} / \mathrm{min}$ ) covering distances at sprint speed of between 337 and $444 \mathrm{~m}$. With regard to the Dutch team, the maximal distance at sprint speed was $283 \mathrm{~m}$ on MD-5. Therefore, it is possible to conclude that the Portuguese team has more volume and intensity in their training session than the Dutch team. This tendency in Dutch teams was also confirmed in a previous study that revealed values between 105 and $281 \mathrm{~m}$ covered at sprint speed (Stevens et al., 2017). Such values only represent $\sim 38 \%$ of sprint running ( $>20 \mathrm{~km} \cdot \mathrm{h}^{-}$ 1) performed $(\sim 730 \mathrm{~m})$ in official Dutch matches (Stevens et al., 2017). On the other hand, a study conducted in a Portuguese team found that a professional team may cover from 500 to $800 \mathrm{~m}$ at high-intensity running to sprint $\left(>18 \mathrm{~km} \cdot \mathrm{h}^{-1}\right)$ during official matches (Silva et al., 2013). The results of our study show that the team may achieve $\sim 440 \mathrm{~m}$ at high sprint running during training sessions, thus representing from 55 to $88 \%$ of the distance covered during matches. Such results reveal that during training sessions of the Dutch team the real effort of the match is replicated to the much greater extent compared to the Portuguese team.

A limitation of the present study was that only one team from each country was analyzed; thus, caution is needed with regard to generalization of the findings. Moreover, a 
comparison between a match and training sessions must be carried out to compare the training stimulus with the match effort. Finally, it would be interesting to compare training methodology and the tasks used in each country to identify what may cause the significant differences in the volume and intensity within training sessions.

Despite the study limitations, this is one of the first studies that has compared external loads of teams from different countries which may promote a research line to characterize the weekly periodization of teams across Europe. The knowledge about the external load in different countries will help understand the differences and the causes for multiple match performances that happen in the game. Understating of the high effort made during training sessions will also help manage the training load and optimize performance of players. The comparison between countries may be useful to identify the best training strategies to be applied in different contexts.

\section{Conclusions}

Greatest distances and intensities were performed on MD-5 and MD-3. The closer proximity to the match days results in a decrease in the training volume, highlighting a conscious tapering period. The Portuguese team covered significantly more distance at higher speed than the Dutch team in the first two days after the match, however, the Dutch team covered significantly more distance at different intensities on the day before the competition. No significant differences between countries were found in the middle phase of the microcycle (i.e. MD-3, MD-4) training session (MD-5), thus suggesting that the greatest load and the acquisition day occur in the middle of the week in both countries. As practical implications, it should be emphasized that different workload profiles and weekly management occurs across the European countries and coaches must be aware of that to prepare players when they come to new clubs and training realities. Moreover, future comparisons with fitness levels and playing style demands will help understand which training approach is more beneficial.

\section{Acknowledgements}

The authors would like to thank for the participation of Francisco Calvete during the experimental protocol. We would like to also thank JOHAN sports for allowing the use of their GPS trackers.

\section{References}

Abade EA, Gonçalves BV, Leite NM, Sampaio JE. Time-Motion and Physiological Profile of Football Training Sessions Performed by Under-15, Under-17, and Under-19 Elite Portuguese Players. Int J Sport Phys Perfor, 2014; 9: 463-470

Akenhead R, Harley JA, Tweddle SP. Examining the External Training Load of an English Premier League Football Team With Special Reference to Acceleration. J Streng Cond Res, 2016; 30: 2424-2432

Anderson L, Orme P, Di Michele R, Close GL, Morgans R, Drust B, Morton JP. Quantification of training load during one-, two- and three-game week schedules in professional soccer players from the English Premier League: implications for carbohydrate periodisation. J Sport Sci, 2016; 34: 1250-1259

Bosquet L, Montpetit J, Arvisais D, Mujika I. Effects of Tapering on Performance. Med Sci Sport Exer, 2007; 39: 1358-1365

Bradley PS, Noakes TD. Match running performance fluctuations in elite soccer: Indicative of fatigue, pacing or situational influences? J Sport Sci, 2013; 31: 1627-1638

Buchheit M, Mendez-Villanueva A, Delhomel G, Brughelli M, Ahmaidi S. Improving repeated sprint ability in young elite soccer players: repeated shuttle sprints vs. explosive strength training. J Streng Cond Res, 2010; 24: 2715-2722

Casamichana D, Castellano J, Dellal A. Influence of Different Training Regimes on Physical and Physiological Demands During Small-Sided Soccer Games. J Streng Cond Res, 2013; 27: 690-697 
Clemente FM, Couceiro MS, Martins FML, Ivanova MO, Mendes R. Activity profiles of soccer players during the 2010 world cup. J Hum Kinet, 2013; 38: 201-11

Clemente FM, Mendes B, Nikolaidis PT, Calvete F, Carriço S, Owen AL. Internal training load and its longitudinal relationship with seasonal player wellness in elite professional soccer. Phys Beh, 2017; 179: 262-267

Clemente FM, Nikolaidis PT. Profile of 1-month training load in male and female football and futsal players. SpringerPlus, 2016; 5: 694

Clemente FM, Nikolaidis PT, Van Der Linden CMIN, Silva B. Effects of Small-Sided Soccer Games on Internal and External Load and Lower Limb Power: A Pilot Study in Collegiate Players. Hum Mov, 2017; 18: 50-57

Coutinho D, Gonçalves B, Figueira B, Abade E, Marcelino R, Sampaio J. Typical weekly workload of under 15, under 17, and under 19 elite Portuguese football players. J Sport Sci, 2015; 33: 1229-37

Cummins C, Orr R, O'Connor H, West C. Global Positioning Systems (GPS) and Microtechnology Sensors in Team Sports: A Systematic Review. Sports Med, 2013; 43: 1025-1042

Dellal A, Keller D, Carling C, Chaouachi A, Wong DP, Chamari K. Physiologic Effects of Directional Changes in Intermittent Exercise in Soccer Players. J Streng Cond Res, 2010; 24: 3219-3226

Di Salvo V, Baron R, Tschan H, Calderon Montero FJ, Bachl N, Pigozzi F. Performance characteristics according to playing position in elite soccer. Int J Sports Med, 2007; 28: 222-227

Dwyer DB, Gabbett TJ. Global Positioning System Data Analysis: Velocity Ranges and a New Definition of Sprinting for Field Sport Athletes. J Streng Cond Res, 2012; 26: 818-824

Ferguson CJ. An effect size primer: A guide for clinicians and researchers. Professional Psychology: Res Practice, 2009; 40: 532-538

Fessi MS, Zarrouk N, Di Salvo V, Filetti C, Barker AR, Moalla W. Effects of tapering on physical match activities in professional soccer players. J Sport Sci, 2016; 34: 2189-2194

Foster C, Florhaug JA, Franklin J, Gottschall L, Hrovatin LA, Parker S, Doleshal P, Dodge C. A new approach to monitoring exercise training. J Streng Cond Res, 2001; 15: 109-115

Gamble P. Strength and conditioning for team sports: Sport-specific physical preparation for high performance. New York, USA: Routledge; 2013

Gamble, P. Training for Sports Speed and Agility: An Evidence-based Approach. Oxon, United Kingdom: Routledge; 2012

Impellizzeri FM, Rampinini E, Coutts AJ, Sassi A, Marcora SM. Use of RPE-based training load in soccer. Med Sci Sport Exer, 2004; 36: 1042-1047

Issurin VB. New horizons for the methodology and physiology of training periodization. Sport Med, 2010; 40: 189-206

Jeong TS, Reilly T, Morton J, Bae SW, Drust B. Quantification of the physiological loading of one week of "pre-season" and one week of "in-season" training in professional soccer players. J Sport Sci, 2011; 29: $1161-1166$

Los Arcos A, Mendez-Villanueva A, Martínez-Santos R. In-season training periodization of professional soccer players. Biol of Sport, 2017; 2: 149-155

Malone JJ, Di Michele R, Morgans R, Burgess D, Morton JP, Drust B. Seasonal Training-Load Quantification in Elite English Premier League Soccer Players. Int J Sport Phys Perfor, 2015; 10: 489-497

Manzi V, D'Ottavio S, Impellizzeri FM, Chaouachi A, Chamari K, Castagna C. Profile of weekly training load in elite male professional basketball players. J Streng Cond Res, 2010; 24: 1399-1406

Mendez-Villanueva A, Buchheit M, Simpson B, Bourdon P. Match Play Intensity Distribution in Youth Soccer. Int J Sport Med, 2012; 34: 101-110

Mohr M, Krustrup P, Bangsbo J. Match performance of high-standard soccer players with special reference to development of fatigue. J Sport Sci, 2003; 21: 519-528

O'Donoghue P. Statistics for sport and exercise studies: An introduction. Book, London and New York, UK and

(C) Editorial Committee of Journal of Human Kinetics 
USA: Routledge Taylor \& Francis Group; 2012

Owen A, Cossio-Bolaños M, Dunlop G, Rouissi M, Chtara M, Bragazzi N, Chamari K. Stability in postseasonal hematological profiles in response to high competitive match-play loads within elite top level European soccer players: implications from a pilot study. Open Access J Sport Med, n.d., ahead-of-print

Owen AL, Djaoui L, Newton M, Malone S, Mendes B. (2017). A contemporary multi-modal mechanical approach to training monitoring in elite professional soccer. Sci Med Football, 2017; 1: 216-221

Owen AL, Wong PL. In-season weekly high-intensity training volume among professional English soccer players: A 20-week study. Soccer J, 2009; March-Apri: 28-32

Rampinini E, Bosio A, Ferraresi I, Petruolo A, Morelli A, Sassi A. Match-Related Fatigue in Soccer Players. Med Sci Sport Exer, 2011; 43: 2161-2170

Rampinini E, Coutts AJ, Castagna C, Sassi R, Impellizzeri FM. Variation in top level soccer match performance. Int J Sport Med, 2007; 28: 1018-1024

Scott MTU, Scott TJ, Kelly VG. The Validity and Reliability of Global Positioning Systems in Team Sport. J Streng Cond Res, 2016; 30: 1470-1490

Silva JR, Magalhães J, Ascensão A, Seabra AF, Rebelo AN. Training Status and Match Activity of Professional Soccer Players Throughout a Season. J Streng Cond Res, 2013; 27: 20-30

Stevens TGA, de Ruiter CJ, Twisk JWR, Savelsbergh GJP, Beek PJ. Quantification of in-season training load relative to match load in professional Dutch Eredivisie football players. Sci Med Football, 2017; 1: 117125

Wrigley R, Drust B, Stratton G, Scott M, Gregson W. Quantification of the typical weekly in-season training load in elite junior soccer players. J Sport Sci, 2012; 30: 1573-1580

\section{Corresponding author:}

\section{Filipe Manuel Clemente}

Complexo Desportivo e Lazer de Melgaço - Monte de Prado, 4960-320, Melgaço, Portugal;

Tel.: 00351258809678

E-mail: filipe.clemente5@gmail.com 